\title{
Biomarkers and phenotypic expression in Alzheimer's disease: exploring the contribution of frailty in the Alzheimer's Disease Neuroimaging Initiative
}

\author{
Marco Canevelli $(\mathbb{D})$ Ivan Arisi • Ilaria Bacigalupo • \\ Andrea Arighi • Daniela Galimberti • Nicola \\ Vanacore • Mara D'Onofrio • Matteo Cesari • \\ Giuseppe Bruno • \\ for the Alzheimer's Disease Neuroimaging Initiative
}

Received: 6 August 2020 / Accepted: 23 October 2020 / Published online: 19 November 2020

(C) The Author(s) 2020

\begin{abstract}
The present study aimed at investigating if the main biomarkers of Alzheimer's disease (AD) neuropathology and their association with cognitive disturbances and dementia are modified by the individual's frailty status. We performed a cross-sectional analysis of data from participants with normal cognition, mild
\end{abstract}

Data used in preparation of this article were obtained from the Alzheimer's Disease Neuroimaging Initiative (ADNI) database (adni.loni.usc.edu). As such, the investigators within the ADNI contributed to the design and implementation of ADNI and/or provided data but did not participate in analysis or writing of this report. A complete listing of ADNI investigators can be found at: http://adni.loni.usc.edu/wp-content/uploads/how_to

apply/ADNI_Acknowledgement_List.pdf.

M. Canevelli $(\bowtie) \cdot$ G. Bruno

Department of Human Neuroscience, "Sapienza" University of

Rome, Rome, Italy

e-mail: marco.canevelli@uniroma1.it

M. Canevelli • I. Bacigalupo $\cdot$ N. Vanacore

National Center for Disease Prevention and Health Promotion, Italian National Institute of Health, Rome, Italy

I. Arisi

Bioinformatics Facility, European Brain Research Institute (EBRI)

Rita Levi-Montalcini, Rome, Italy

I. Arisi · M. D’Onofrio

Institute of Translational Pharmacology (IFT), CNR, Rome, Italy

A. Arighi · D. Galimberti

Neurodegenerative Diseases Unit, Fondazione IRCCS Ca' Granda

Ospedale Maggiore Policlinico, Milan, Italy cognitive impairment (MCI), and AD dementia enrolled in the Alzheimer's Disease Neuroimaging Initiative 2 (ADNI2) study. Frailty was operationalized by computing a 40-item Frailty Index (FI). The following AD biomarkers were considered and analyzed according to the participants' frailty status: $\mathrm{CSF} A \beta_{1-42},{ }^{181} \mathrm{P}$-tau, and

\footnotetext{
A. Arighi · D. Galimberti

Dino Ferrari Center, University of Milan, Milan, Italy

D. Galimberti

Department of Biomedical, Surgical and Dental Sciences,

University of Milan, Milan, Italy

M. D’Onofrio

Genomics Facility, European Brain Research Institute (EBRI) Rita Levi-Montalcini, Rome, Italy

M. Cesari

Department of Clinical Sciences and Community Health,

University of Milan, Milan, Italy

M. Cesari

Geriatric Unit, IRCCS Istituti Clinici Scientifici Maugeri, Milan, Italy
} 
T-tau; MRI-based hippocampus volume; cortical glucose metabolism at the FDG PET imaging; amyloid deposition at the ${ }^{18} \mathrm{~F}-\mathrm{AV}-45$ PET imaging. Logistic regression models, adjusted for age, sex, and education, were performed to explore the association of biomarkers with cognitive status at different FI levels. Subjects with higher FI scores had lower CSF levels of $A \beta_{1-42}$, hippocampus volumes at the MRI, and glucose metabolism at the FDG PET imaging, and a higher amyloid deposition at the ${ }^{18} \mathrm{~F}-\mathrm{AV}-45$ PET. No significant differences were observed among the two frailty groups concerning ApoE genotype, CSF T-tau, and P-tau. Increasing frailty levels were associated with a weakened relationship between dementia and ${ }^{18} \mathrm{~F}-\mathrm{AV}-45$ uptake and hippocampus volume and with a stronger relationship of dementia with FDG PET. Frailty contributes to the discrepancies between AD pathology and clinical manifestations and influences the association of $\mathrm{AD}$ pathological modifications with cognitive changes. $\mathrm{AD}$ and dementia should increasingly be conceived as "complex diseases of aging," determined by multiple, simultaneous, and interacting pathophysiological processes.

Keywords Alzheimer's disease · Frailty · Biomarkers · Aging $\cdot$ Dementia

\section{Introduction}

The relationship between the biological modifications underlying Alzheimer's disease (AD) and their phenotypic expression is highly complex. A non-negligible proportion of people diagnosed with $\mathrm{AD}$ has few neuropathological abnormalities at autopsy, whereas many cognitively intact individuals exhibit a high burden of AD pathology [1]. Accordingly, a relevant discordance between biomarker- and clinical-based definitions of $\mathrm{AD}$ has repeatedly been documented [2]. The understanding of this relationship may be improved by the adoption of constructs and models that comprehensively reflect the biological complexity of the organism as well as the heterogeneity of health trajectories and outcomes within aging. In this regard, the concept of frailty may open promising scenarios in the field.

Frailty is intended as a condition characterized by reduced homeostatic reserves and increased vulnerability to stressors exposing the individual to adverse outcomes $[3,4]$. This construct has triggered growing attention in many medical areas [5] as it likely contributes to the relevant variability of health outcomes. Furthermore, it may affect the health trajectories of individuals presenting similar risk profiles (e.g., diagnosed with the same disease) [6].

Frailty is frequently operationalized using a deficit accumulation approach [7]. According to this model, the individual's degree of frailty is related to the extent of the health deficits he/she has accumulated during the life course. The one's biological complexity and risk profile can therefore be estimated by quantifying (i.e., arithmetically counting) these negative attributes and condensing them in a single continuous variable, the so-called Frailty Index (FI) [6].

Frailty as accumulation of deficits has already been investigated in the field of dementia and cognitive disorders. It has been shown to independently predict incident dementia among cognitively normal older individuals $[8,9]$. Increasing FI scores have been associated with a higher probability of conversion to dementia in subjects with mild cognitive impairment (MCI), and poorer outcomes (i.e., mortality, hospitalization, and steeper worsening of cognitive functioning) in patients with AD [10-12]. Moreover, the FI has been adopted to ascertain the external validity of research protocols enrolling participants with dementia [13]. There is also emerging evidence that frailty, quantified as FI, can influence the neuropathological and biological changes occurring with brain aging and neurodegeneration and the relationship with their phenotypic manifestations $[14,15]$. In particular, in a recent analysis of the clinicopathological data from a large sample of communitydwelling older adults, frailty was found to moderate the association between neuropathology and dementia in $\mathrm{AD}[15]$.

Based on these premises, it can be hypothesized that the individual's frailty status may modify the association between candidate AD biomarkers, reflecting in vivo the main neuropathological modifications of the disease, and the cognitive manifestations occurring along the AD continuum [16]. Testing this hypothesis could have important implications since the use of biomarkers is becoming crucial for diagnostic purposes as well as for the identification and development of potential therapeutic targets [17-19]. The present cross-sectional study is aimed at investigating if, and eventually how, frailty influences the changes of the main biomarkers of $\mathrm{AD}$ pathology and moderates their relationship with cognitive changes and dementia. 


\section{Methods}

Data sources

Data used in the preparation of this study were obtained from the Alzheimer's Disease Neuroimaging Initiative (ADNI) database (http://adni.loni.usc.edu). The ADNI was launched in 2003 as a public-private partnership, led by Principal Investigator Michael W. Weiner, MD. The primary goal of ADNI has been to test whether serial magnetic resonance imaging (MRI), positron emission tomography (PET), other biological markers, and clinical and neuropsychological assessment can be combined to measure the progression of MCI and early $\mathrm{AD}$ (for up-to-date information, see http://adni.loni.usc. edu).

Participants and procedures

Data from 778 eligible participants in the ADNI2 study (phase 2 of the ADNI project) were considered for the present analysis. Subjects enrolled in the study were categorized into four diagnostic categories according to their cognitive and functional status:

(i) cognitively normal $(\mathrm{CN})$;

(ii) early $\mathrm{MCI}(\mathrm{EMCI})$;

(iii) late $\mathrm{MCI}(\mathrm{LMCI})$;

(iv) mild $\mathrm{AD}$ dementia.

The diagnosis of MCI was based on the Petersen criteria $[20,21]$ and required Mini-Mental State Examination (MMSE) scores between 24 and 30 (inclusive), a subjective memory concern, abnormal memory function documented by education-adjusted scores on the Logical Memory II subscale from the Wechsler Memory Scale (WMS)-Revised [22], a clinical dementia rating of 0.5 , absence of significant impairment in other cognitive domains, globally preserved activities of daily living, and absence of dementia. All enrolled MCI subjects were, thus, amnestic MCI. For the present purposes, participants with EMCI and LMCI, differing for the degree of memory impairment at the WMS, were grouped together in the MCI category. Patients with $\mathrm{AD}$ dementia met the National Institute of Neurological and Communicative Disorders and Stroke and the Alzheimer's Disease and Related Disorders Association criteria for probable AD [23]. The detailed eligibility and diagnostic criteria can be found in the ADNI2 protocol (http://adni.loni.usc.edu/wpcontent/uploads/2008/07/adni2-procedures-manual. pdf).

For each of the considered subjects, data concerning the following domains were downloaded from the ADNI database: demographics; past medical history and comorbidities; general and neurological examination; global cognitive performance, as measured by the MMSE; functional independence, by means of the Functional Assessment Questionnaire (FAQ); apolipoprotein $\mathrm{E}$ (ApoE) genotype; cerebrospinal fluid (CSF) concentrations of amyloid beta $\left(\mathrm{A} \beta_{1-42},\right),{ }^{181}$ phosphotau (P-tau), and total tau (T-tau); MRI-based measurement of hippocampus volumes; cortical glucose metabolism at the fluorodeoxyglucose (FDG) PET imaging; amyloid deposition as measured by the uptake of ${ }^{18} \mathrm{~F}$ AV-45 (i.e., florbetapir) at the PET imaging. All this information referred to the assessments performed during the screening and baseline visits, separated by a maximum window of 28 days.

The detailed description of the different diagnostic procedures, protocols, and measurements is available in the ADNI manual (http://adni.loni.usc.edu) and previous publications [24-27]. The choice of the biomarkers of interest was based on the currently proposed frameworks for the biological definition of AD [18].

\section{Frailty Index}

A FI was operationalized from health variables collected at the screening and baseline visits, following a standard procedure [28]. Candidate variables were incorporated in the FI if they individually met the following criteria:

(i) they must represent health-related deficits, such as symptoms, signs, diseases, and functional impairments, all associated with negative outcomes;

(ii) their prevalence must generally increase with advancing chronological age;

(iii) they must not saturate too early or too late (i.e., they should not be present in $>80 \%$ or in $<1 \%$ of the study population, respectively);

(iv) they must contain $<5 \%$ missing values.

Moreover, the identified set of deficits must also respond to some overall requirements such as covering multiple organ systems and grouping at least 30 variables. Based on the study aims, variables strongly 
related to dementia and cognitive status were not considered in the computation. The 40 deficits included in the present FI are listed in Table 1. Each variable was coded as 0 (i.e., absent or normal) or 1 (i.e., present or abnormal). The ability to perform daily activities, as measured by the FAQ, was instead more finely graded (i.e., "normal" $=0$; "has difficulty but does by self" = 0.25 ; "requires assistance" $=0.5$; "dependent" $=1$ ).

The FI was calculated by dividing the sum of deficits presented by the individual divided for the number of considered deficits (i.e., 40). For instance, a participant presenting 10 out of the 40 deficits had a resulting FI of 0.25 (i.e., 10/40).

The variables needed to compute the 40 -item FI were available for 778 out of the 789 participants to the ADNI2 protocol.

\section{Statistical analysis}

Descriptive analyses were conducted to present the characteristics of the study sample. Participants were categorized into two groups according to their frailty status, using the median value of the FI as the cut-point. Unpaired two-sided heteroscedastic $T$-tests or one-way ANOVA (for continuous variables) and two-sided chisquare tests (for categorical variables) were performed to compare the characteristics of participants and biomarkers by frailty and cognitive status. Spearman's correlation coefficients were calculated to assess the strength and direction of the correlation between FI and age. For each of the considered biomarkers, two levels (i.e., low or high) were created adopting the median as the cut-point.

Biomarkers resulting as significantly different between the two frailty groups at the univariate analysis were then included as binary independent variables in logistic regression models exploring their association with cognitive status (i.e., AD or MCI vs. $\mathrm{CN}$ ) (binary dependent variable) at the two levels of FI. Interaction (on the multiplicative scale) between FI and each biomarker in their association with cognitive status was calculated as the ratio between the odds ratio (OR) of association between biomarkers and cognitive status in the high FI group and the OR of association in the low FI group. That is, $\mathrm{OR}_{\text {interaction }}=\mathrm{OR}_{\mathrm{highFI}} / \mathrm{OR}_{\text {lowFI }}[29]$. All models were adjusted for age (continuous variable), sex (binary variable), and education (continuous variable). Sensitivity analyses were conducted using: (i) a modified 32-item FI not incorporating functional deficits (i.e., items 33 to 40 of the original FI) to avoid possible overlaps with diagnostic classifications (i.e., MCI and dementia); and (ii) different cutoffs that had been previously adopted in the ADNI study to classify the ${ }^{18} \mathrm{~F}-\mathrm{AV}-45$, CSF $\mathrm{A} \beta_{1-42}$, FDG, and hippocampus volume status [30-32]. ApoE status was not included as a confounder in the models because not significantly different in the two frailty groups at the univariate analysis.

The accuracy of the median FI score at detecting dementia was estimated by the area under the ROC curve (AUC). Specifically, the sensitivity, specificity, and AUC for the median FI cut-point were calculated .

The level of statistical significance was set at $p<$ 0.05 . All analyses were performed using SPSS version 25 for Mac.

\section{Results}

Overall, $291 \mathrm{CN}$ subjects, 338 participants with MCI, and 149 patients with mild AD dementia were considered in the present study. Their sociodemographic and clinical characteristics are summarized in Tables 2 and 3.

The FI had a characteristic right-skewed distribution, both in the whole sample and in each of the three diagnostic groups (Fig. 1), and ranged between 0 and 0.56. The median FI score was 0.20 (IQR $=0.14-0.27$ ) and the 99th percentile was 0.44 .

The FI exhibited a statistically significant correlation with participants' chronological age (Spearman's rho = $0.20 ; p<0.001)$. Subjects with higher FI values (i.e., > $0.20 ; n=366$ ) were significantly older, less educated, more frequently male, more likely to have a diagnosis of dementia, and had lower MMSE scores compared to those with a FI score lower than the median value (Table 3). Moreover, they had lower CSF levels of $\mathrm{A} \beta_{1-42}$, hippocampus volumes at the MRI, and glucose metabolism at the FDG PET imaging, and a higher amyloid deposition at the ${ }^{18} \mathrm{~F}-\mathrm{AV}-45$ PET. Conversely, no significant differences were observed among the two frailty groups concerning ApoE genotype, and CSF levels of T-tau and P-tau (Table 3).

A limited concordance was observed between $\mathrm{AD}$ biomarkers and the cognitive status of participants in the overall sample. Indeed, a proportion ranging between 19.8 and $22.8 \%$ of those with an abnormal biomarker status (i.e., CSF $A \beta_{1-42}$, hippocampus volume, and 
Table 1 Deficits considered in the computation of the Frailty Index

\begin{tabular}{|c|c|c|c|c|}
\hline \multirow[t]{2}{*}{ Items } & \multicolumn{4}{|l|}{ Scoring } \\
\hline & 0 & 0.25 & 0.5 & 1 \\
\hline 1. Renal-genitourinary diseases & No & - & - & Yes \\
\hline 2. Dermatologic-connective diseases & No & - & - & Yes \\
\hline 3. Hepatic diseases & No & - & - & Yes \\
\hline 4. Cardiovascular diseases & No & - & - & Yes \\
\hline 5. Endocrine-metabolic diseases & No & - & - & Yes \\
\hline 6. Neurological (non-AD) diseases & No & - & - & Yes \\
\hline 7. Psychiatric diseases & No & - & - & Yes \\
\hline 8. Malignancies & No & - & - & Yes \\
\hline 9. Musculoskeletal diseases & No & - & - & Yes \\
\hline 10. Gastrointestinal diseases & No & - & - & Yes \\
\hline 11. Respiratory diseases & No & - & - & Yes \\
\hline 12. Head, eyes, ears, nose, and throat diseases & No & - & - & Yes \\
\hline 13. Hematopoietic-lymphatic diseases & No & - & - & Yes \\
\hline 14. Urinary discomfort & Absent & - & - & Present \\
\hline 15. Shortness of breath & Absent & - & - & Present \\
\hline 16. Low energy & Absent & - & - & Present \\
\hline 17. Falls & Absent & - & - & Present \\
\hline 18. Insomnia & Absent & - & - & Present \\
\hline 19. Constipation & Absent & - & - & Present \\
\hline 20. Drowsiness & Absent & - & - & Present \\
\hline 21. Dizziness & Absent & - & - & Present \\
\hline 22. Musculoskeletal pain & Absent & - & - & Present \\
\hline 23. Seated BP diastolic & $\leq 90 \mathrm{mmHg}$ & - & - & $>90 \mathrm{mmHg}$ \\
\hline 24. Seated BP systolic & $\leq 140 \mathrm{mmHg}$ & - & - & $>140 \mathrm{mmHg}$ \\
\hline 25. Tremor & Absent & - & - & Present \\
\hline 26. Motor strength & Normal & - & - & Abnormal \\
\hline 27. Gait & Normal & - & - & Abnormal \\
\hline 28. Cerebellar - finger to nose & Normal & - & - & Abnormal \\
\hline 29. Agitation/aggression (NPI) & No & - & - & Yes \\
\hline 30. Anxiety (NPI) & No & - & - & Yes \\
\hline 31. Apathy/indifference (NPI) & No & - & - & Yes \\
\hline 32. Irritability/lability (NPI) & No & - & - & Yes \\
\hline 33. Heating water, making a cup of coffee (FAQ) & 0 & 1 & 2 & 3 \\
\hline 34. Traveling out of the neighborhood (FAQ) & 0 & 1 & 2 & 3 \\
\hline 35. Preparing a balanced meal (FAQ) & 0 & 1 & 2 & 3 \\
\hline 36. Writing checks, paying bills, or balancing checkbook (FAQ) & 0 & 1 & 2 & 3 \\
\hline 37. Paying attention to and understanding a TV program, book, or magazine (FAQ) & 0 & 1 & 2 & 3 \\
\hline 38. Playing a game of skill such as bridge or chess (FAQ) & 0 & 1 & 2 & 3 \\
\hline 39. Shopping alone for clothes, household (FAQ) & 0 & 1 & 2 & 3 \\
\hline 40. Assembling tax records, business affairs (FAQ) & 0 & 1 & 2 & 3 \\
\hline
\end{tabular}

FAQ Functional Assessment Questionnaire, NPI Neuropsychiatric Inventory 
Table 2 Sociodemographic and clinical characteristics of participants according to their cognitive status

\begin{tabular}{|c|c|c|c|c|}
\hline & $\mathrm{CN}(n=291)$ & $\operatorname{MCI}(n=338)$ & AD dementia $(n=149)$ & $p$ \\
\hline \multicolumn{5}{|l|}{ Age (years) } \\
\hline $\begin{array}{l}\text { Mean } \pm \text { SD } \\
\text { Median (IQR) }\end{array}$ & $\begin{array}{l}73.0 \pm 6.0 \\
72.5(68.3-77.0)\end{array}$ & $\begin{array}{l}71.6 \pm 7.4 \\
71.9(66.4-76.8)\end{array}$ & $\begin{array}{l}74.6 \pm 8.2 \\
75.2(70.4-80.2)\end{array}$ & $<0.001$ \\
\hline Women, $n(\%)$ & $157(54.0)$ & $153(45.3)$ & $62(41.6)$ & 0.023 \\
\hline \multicolumn{5}{|l|}{ Education (years) } \\
\hline $\begin{array}{l}\text { Mean } \pm \mathrm{SD} \\
\text { Median }(\mathrm{IQR})\end{array}$ & $\begin{array}{l}16.6 \pm 2.5 \\
16.0(15.0-18.0)\end{array}$ & $\begin{array}{l}16.3 \pm 0.1 \\
16.0(14.0-18.0)\end{array}$ & $\begin{array}{l}15.8 \pm 2.7 \\
16.0(14.0-18.0)\end{array}$ & 0.009 \\
\hline \multicolumn{5}{|l|}{ Frailty Index } \\
\hline $\begin{array}{l}\text { Mean } \pm \mathrm{SD} \\
\text { Median }(\mathrm{IQR})\end{array}$ & $\begin{array}{l}0.18 \pm 0.08 \\
0.18(0.13-0.23)\end{array}$ & $\begin{array}{l}0.21 \pm 0.09 \\
0.20(0.15-0.28)\end{array}$ & $\begin{array}{l}0.26 \pm 0.09 \\
0.26(0.20-0.33)\end{array}$ & $<0.001$ \\
\hline \multicolumn{5}{|l|}{ MMSE } \\
\hline $\begin{array}{l}\text { Mean } \pm \mathrm{SD} \\
\text { Median }(\mathrm{IQR})\end{array}$ & $\begin{array}{l}29.0 \pm 1.2 \\
29.0(29.0-30.0)\end{array}$ & $\begin{array}{l}28.0 \pm 1.7 \\
28.0(27.0-29.0)\end{array}$ & $\begin{array}{l}23.1 \pm 2.1 \\
23.0(21.0-25.0)\end{array}$ & $<0.001$ \\
\hline \multicolumn{4}{|l|}{$\mathrm{ApoE} \varepsilon 4, n(\%)$} & \multirow[t]{2}{*}{$<0.001$} \\
\hline $\begin{array}{l}1 \varepsilon 4 \text { allele } \\
2 \varepsilon 4 \text { allele }\end{array}$ & $\begin{array}{l}81(27.9) \\
7(2.4)\end{array}$ & $\begin{array}{l}131(39.1) \\
38(11.3)\end{array}$ & $\begin{array}{l}69(47.6) \\
28(19.3)\end{array}$ & \\
\hline \multicolumn{5}{|c|}{$\operatorname{CSF} \mathrm{A} \beta_{1-42}(\mathrm{pg} / \mathrm{ml})$} \\
\hline $\begin{array}{l}\text { Mean } \pm \text { SD } \\
\text { Median }(I Q R)\end{array}$ & $\begin{array}{l}1237.0 \pm 437.5 \\
1306.0(867.5-1700.0)\end{array}$ & $\begin{array}{l}980.7 \pm 413.2 \\
876.7(664.7-1293.0)\end{array}$ & $\begin{array}{l}691.8 \pm 322.0 \\
622.5(489.4-785.8)\end{array}$ & $<0.001$ \\
\hline \multicolumn{5}{|c|}{ CSF T-tau (pg/ml) } \\
\hline $\begin{array}{c}\text { Mean } \pm \text { SD } \\
\text { Median (IQR) } \\
\text { CSF }{ }^{181} \text { P-tau (pg) }\end{array}$ & $\begin{array}{l}238.4 \pm 92.4 \\
213.9(174.4-298.2)\end{array}$ & $\begin{array}{l}280.0 \pm 134.0 \\
247.5(186.5-331.5)\end{array}$ & $\begin{array}{l}373.1 \pm 153.2 \\
331.5(266.8-445.4)\end{array}$ & $<0.001$ \\
\hline $\begin{array}{l}\text { Mean } \pm \mathrm{SD} \\
\text { Median }(\mathrm{IQR})\end{array}$ & $\begin{array}{l}21.8 \pm 9.4 \\
19.3(15.3-26.3)\end{array}$ & $\begin{array}{l}27.0 \pm 14.9 \\
23.2(16.8-33.0)\end{array}$ & $\begin{array}{l}36.8 \pm 16.0 \\
33.2(25.2-45.5)\end{array}$ & $<0.001$ \\
\hline \multicolumn{5}{|c|}{ MRI hippocampus (ml) } \\
\hline $\begin{array}{c}\text { Mean } \pm \mathrm{SD} \\
\text { Median }(\mathrm{IQR}) \\
\text { FDG PET (metaR }\end{array}$ & $\begin{array}{l}7.5 \pm 0.9 \\
7.5(7.0-8.0)\end{array}$ & $\begin{array}{l}7.0 \pm 1.1 \\
7.0(6.3-7.8)\end{array}$ & $\begin{array}{l}5.9 \pm 9.7 \\
5.8(5.2-6.6)\end{array}$ & $<0.001$ \\
\hline $\begin{array}{c}\text { Mean } \pm \text { SD } \\
\text { Median }(\mathrm{IQR}) \\
{ }^{18} \text { F-AV-45 (SUV }\end{array}$ & $\begin{array}{l}1.32 \pm 0.11 \\
1.32(1.25-1.39)\end{array}$ & $\begin{array}{l}1.25 \pm 0.13 \\
1.25(1.17-1.33)\end{array}$ & $\begin{array}{l}1.07 \pm 0.15 \\
1.07(0.98-1.17)\end{array}$ & $<0.001$ \\
\hline $\begin{array}{l}\text { Mean } \pm \text { SD } \\
\text { Median (IQR) }\end{array}$ & $\begin{array}{l}1.12 \pm 0.18 \\
1.06(1.00-1.19)\end{array}$ & $\begin{array}{l}1.23 \pm 0.23 \\
1.19(1.02-1.40)\end{array}$ & $\begin{array}{l}1.40 \pm 0.22 \\
1.43(1.27-1.54)\end{array}$ & $<0.001$ \\
\hline
\end{tabular}

$\overline{A D}$ Alzheimer's disease, $A p o E$ apolipoprotein E, ${ }^{18} F-A V-45$ florbetapir, $C N$ cognitively normal, $C S F$ cerebrospinal fluid, $F D G$ fluorodeoxyglucose (18F), MCI mild cognitive impairment, MMSE Mini-Mental State Examination, MRI magnetic resonance imaging, PET positron emission tomography

Missing data: ApoE genotype: $n=8$; CSF A $\beta_{1-42}: n=82$; CSF T-tau: $n=82$; CSF ${ }^{181}$ P-tau: $n=82$; MRI hippocampus: $n=87$; FDG PET: $n$ $=13 ;{ }^{18} \mathrm{~F}-\mathrm{AV}-45: n=22$. The statistical significance was computed by the two-sided chi-square test for sex and ApoE genotype; by one-way ANOVA otherwise

FDG uptake values lower than median cut-points, ${ }^{18} \mathrm{~F}$ AV-45 uptake higher than the median value) had normal cognitive functioning. On the other hand, a relevant proportion of those with normal biomarkers, varying between 45.0 and $46.6 \%$, met the criteria for MCI or mild AD dementia (Table 4). Among participants with abnormal biomarker values, the prevalence of dementia was higher in those with higher FI scores compared to those with $\mathrm{FI} \leq 0.20$ (i.e., $42.9 \%$ vs. $18.7 \%$ for high ${ }^{18} \mathrm{~F}$ AV-45 uptake; $43.1 \%$ vs. $19.2 \%$ for low CSF A $\beta_{1-42}$; $42.9 \%$ vs. $17.5 \%$ for low hippocampus volume; $46.7 \%$ vs. 19.1 for low FDG uptake). Conversely, in the case of normal biomarker status, the proportion of $\mathrm{CN}$ cases was higher in those with lower FI scores (i.e., 62.9\% vs. $41.7 \%$ for low ${ }^{18} \mathrm{~F}-\mathrm{AV}-45$ uptake; $59.3 \%$ vs. $43.9 \%$ for high CSF $A \beta_{1-42} ; 61.3 \%$ vs. $43.0 \%$ for high 
Table 3 Sociodemographic and clinical characteristics of participants according to their frailty status

\begin{tabular}{|c|c|c|c|c|}
\hline & Overall $(n=778)$ & $\mathrm{FI} \leq 0.20(n=412)$ & FI $>0.20(n=366)$ & $p$ \\
\hline \multicolumn{5}{|l|}{ Age (years) } \\
\hline $\begin{array}{l}\text { Mean } \pm \text { SD } \\
\text { Median (IQR) }\end{array}$ & $\begin{array}{l}72.7 \pm 7.2 \\
72.7(67.7-77.6)\end{array}$ & $\begin{array}{l}71.6 \pm 6.7 \\
71.3(66.9-76.2)\end{array}$ & $\begin{array}{l}73.9 \pm 7.5 \\
73.9(68.9-79.1)\end{array}$ & $<0.001$ \\
\hline $\begin{array}{l}\text { Sex, } n(\%) \\
\text { Women } \\
\text { Men }\end{array}$ & $\begin{array}{l}372(47.8) \\
406(52.2)\end{array}$ & $\begin{array}{l}212(51.5) \\
200(48.5)\end{array}$ & $\begin{array}{l}160(43.7) \\
206(56.3)\end{array}$ & 0.03 \\
\hline \multicolumn{5}{|l|}{ Education (years) } \\
\hline $\begin{array}{l}\text { Mean } \pm \text { SD } \\
\text { Median (IQR) } \\
\text { MMSE }\end{array}$ & $\begin{array}{l}16.3 \pm 2.6 \\
16.0(14.0-18.0)\end{array}$ & $\begin{array}{l}16.6 \pm 2.5 \\
16.0(15.0-18.0)\end{array}$ & $\begin{array}{l}16.0 \pm 2.8 \\
16.0(14.0-18.0)\end{array}$ & $<0.01$ \\
\hline $\begin{array}{l}\text { Mean } \pm \text { SD } \\
\text { Median (IQR) }\end{array}$ & $\begin{array}{l}27.4 \pm 2.7 \\
28.0(26.0-30.0)\end{array}$ & $\begin{array}{l}28.1 \pm 2.1 \\
29.0(27.0-30.0)\end{array}$ & $\begin{array}{l}26.7 \pm 3.1 \\
28.0(25.0-29.0)\end{array}$ & $<0.001$ \\
\hline Diagnosis, $n(\%)$ & & & & $<0.001$ \\
\hline $\begin{array}{l}\mathrm{CN} \\
\mathrm{MCI}\end{array}$ & $\begin{array}{l}291(37.4) \\
338(43.4)\end{array}$ & $\begin{array}{l}200(48.5) \\
172(41.8)\end{array}$ & $\begin{array}{l}91(24.9) \\
166(45.3)\end{array}$ & \\
\hline AD dementia & 149 (19.2) & $40(9.7)$ & $109(29.8)$ & \\
\hline $\begin{array}{l}\text { ApoE genotype, } n(\%) \\
1 \varepsilon 4 \text { allele } \\
2 \varepsilon 4 \text { allele }\end{array}$ & $\begin{array}{l}281(36.5) \\
73(9.5)\end{array}$ & $\begin{array}{l}143(34.9) \\
35(8.5)\end{array}$ & $\begin{array}{l}138(38.3) \\
38(10.6)\end{array}$ & 0.28 \\
\hline $\mathrm{CSF} A \beta_{1-42}(\mathrm{pg} / \mathrm{ml})$ & & & & \\
\hline $\begin{array}{c}\text { Mean } \pm \text { SD } \\
\text { Median (IQR) } \\
\text { CSF T-tau (pg/ml) }\end{array}$ & $\begin{array}{l}1020.2 \pm 451.1 \\
909.5(652.0-1434.8)\end{array}$ & $\begin{array}{l}1105.2 \pm 447.2 \\
1006.0(715.5-1620.0)\end{array}$ & $\begin{array}{l}925.4 \pm 437.1 \\
785.8(586.9-1230.0)\end{array}$ & $<0.001$ \\
\hline $\begin{array}{c}\text { Mean } \pm \text { SD } \\
\text { Median (IQR) } \\
\text { CSF }{ }^{181} \text { P-tau (pg/ml) }\end{array}$ & $\begin{array}{l}282.3 \pm 133.2 \\
250.4(189.0-340.4)\end{array}$ & $\begin{array}{l}275.0 \pm 128.3 \\
241.0(189.7-321.5)\end{array}$ & $\begin{array}{l}290.4 \pm 138.3 \\
263.9(188.1-359.8)\end{array}$ & 0.13 \\
\hline $\begin{array}{l}\text { Mean } \pm \text { SD } \\
\text { Median (IQR) } \\
\text { MRI hippocampus (ml) }\end{array}$ & $\begin{array}{l}27.0 \pm 14.4 \\
23.4(17.1-32.8)\end{array}$ & $\begin{array}{l}26.2 \pm 14.1 \\
22.3(17.1-31.1)\end{array}$ & $\begin{array}{l}27.8 \pm 14.6 \\
25.3(17.1-34.2)\end{array}$ & 0.13 \\
\hline $\begin{array}{c}\text { Mean } \pm \mathrm{SD} \\
\text { Median (IQR) } \\
\text { FDG PET (metaROI) }\end{array}$ & $\begin{array}{l}7.0 \pm 1.2 \\
7.1(6.2-7.8)\end{array}$ & $\begin{array}{l}7.3 \pm 1.2 \\
7.3(6.6-8.0)\end{array}$ & $\begin{array}{l}6.7 \pm 1.1 \\
6.8(5.9-7.5)\end{array}$ & $<0.001$ \\
\hline $\begin{array}{l}\text { Mean } \pm \text { SD } \\
\text { Median (IQR) } \\
{ }^{18} \text { F-AV-45 PET (SUVR) }\end{array}$ & $\begin{array}{l}1.24 \pm 0.16 \\
1.26(1.15-1.34)\end{array}$ & $\begin{array}{l}1.27 \pm 0.14 \\
1.29(1.20-1.36)\end{array}$ & $\begin{array}{l}1.21 \pm 0.16 \\
1.22(1.11-1.33)\end{array}$ & $<0.001$ \\
\hline $\begin{array}{l}\text { Mean } \pm \text { SD } \\
\text { Median (IQR) }\end{array}$ & $\begin{array}{l}1.22 \pm 0.23 \\
1.15(1.02-1.40)\end{array}$ & $\begin{array}{l}1.19 \pm 0.22 \\
1.10(1.02-1.34)\end{array}$ & $\begin{array}{l}1.25 \pm 0.24 \\
1.24(1.03-1.43)\end{array}$ & $<0.001$ \\
\hline
\end{tabular}

$A D$ Alzheimer's disease, ApoE apolipoprotein E, ${ }^{18} F-A V-45$ florbetapir, $C N$ cognitively normal, $C S F$ cerebrospinal fluid, $F D G$ fluorodeoxyglucose (18F), MCI mild cognitive impairment, MMSE Mini-Mental State Examination, MRI magnetic resonance imaging, PET positron emission tomography

Missing data: ApoE genotype: $n=8$; CSF A $\beta_{1-42}: n=82$; CSF T-tau: $n=82$; CSF ${ }^{181}$ P-tau: $n=82$; MRI hippocampus: $n=87$; FDG PET: $n$ $=13 ;{ }^{18} \mathrm{~F}-\mathrm{AV}-45: n=22$. The statistical significance was computed by the two-sided chi-square test for sex, diagnosis, and ApoE genotype; by the two-sided $T$-test otherwise

hippocampus volume; $61.1 \%$ vs. $45.1 \%$ for high FDG uptake) (Table 4).

The relationship between each $\mathrm{AD}$ biomarker and cognitive status was influenced by frailty levels (Table 5). In particular, increasing frailty levels were associated with a weaker relationship between dementia and ${ }^{18} \mathrm{~F}-\mathrm{AV}-45$ uptake $\left(\mathrm{OR}_{\text {interaction }}=0.58 ; 95 \% \mathrm{CI}\right.$ : $0.37-0.77)$, and hippocampus volume $\left(\mathrm{OR}_{\text {interaction }}=\right.$ 0.86 ; $95 \%$ CI: $0.64-0.95)$. On the contrary, the association of dementia with FDG PET $\left(\mathrm{OR}_{\text {interaction }}=3.86\right.$; 


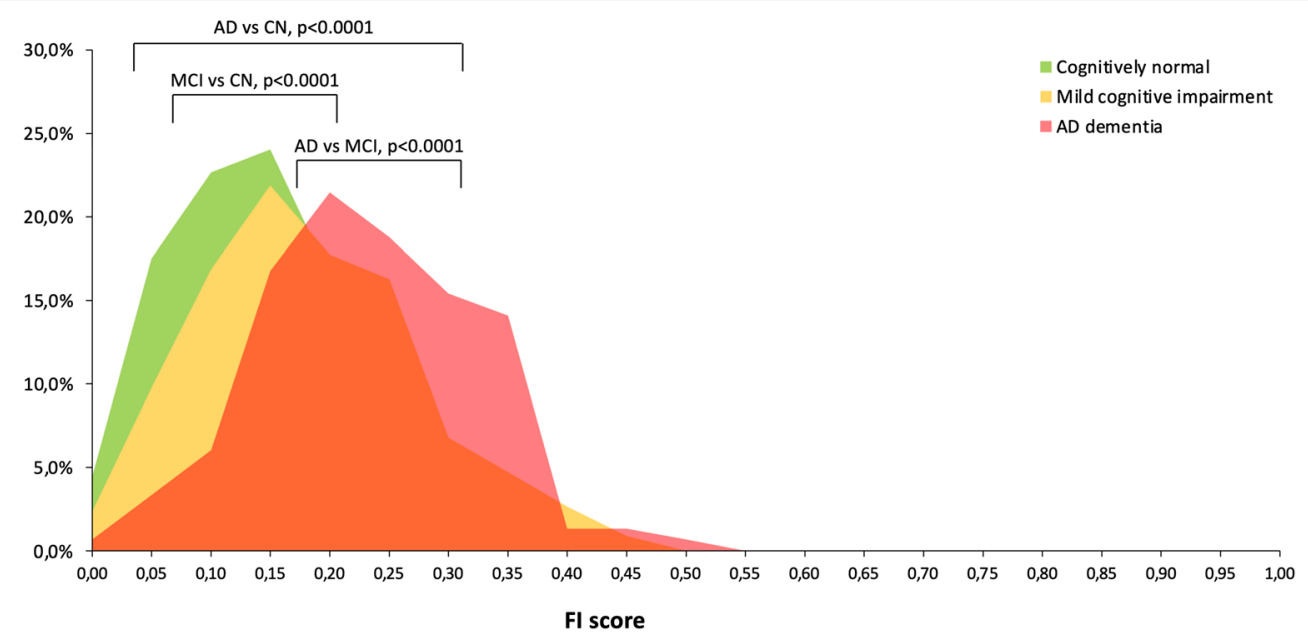

Fig. 1 Distribution of the Frailty Index in the three cognitive groups (cognitively normal: green; mild cognitive impairment: yellow; AD dementia: red). The comparison of the three distributions of the Frailty Index is based on the Kolmogorov-Smirnov two-sided test

95\% CI: 2.37-5.77) was stronger at higher FI scores. Finally, no significant interaction was observed with CSF $A \beta_{1-42}\left(\mathrm{OR}_{\text {interaction }}=1.30 ; 95 \% \mathrm{CI}: 0.93-1.52\right)$. Similar results were obtained when the modified 32item FI, not including functional deficits, was used. Indeed, the increase in FI scores weakened the relationship between dementia and amyloid deposition, and hippocampus volume, while strengthening the relationship between dementia and FDG PET (data not shown). The adoption of the cut-points derived from the official
ADNI protocol and previous studies (i.e., 1.11 SUVR for ${ }^{18} \mathrm{~F}-\mathrm{AV}-45,880 \mathrm{pg} / \mathrm{ml}$ for CSF $\mathrm{A} \beta_{1-42}, 1.21$ metaROI for FDG PET, and $6723 \mathrm{ml}$ for hippocampus volume) did not substantially change the results in sensitivity analyses (Table 6).

As to MCI subjects, only the relationship with ${ }^{18} \mathrm{~F}$ AV-45 was significantly influenced by FI scores $\left(\mathrm{OR}_{\text {interaction }}=0.85 ; 95 \% \mathrm{CI}: 0.37-0.98\right)($ Table 5$)$.

ROC curve analysis (AUC $0.71 ; 95 \%$ CI $0.67-0.75$, $p<0.001$ ) showed that the adopted FI cut-point (i.e.,

Table 4 Proportion of participants with normal cognition, MCI, or AD dementia according to Frailty Index values by each of the considered biomarkers. Data are shown as $n(\%)$

\begin{tabular}{|c|c|c|c|c|c|c|c|c|c|c|}
\hline & & \multicolumn{6}{|c|}{ Frailty Index } & & & \\
\hline & & \multicolumn{3}{|l|}{ Low } & \multicolumn{3}{|l|}{ High } & \multicolumn{3}{|l|}{ Total } \\
\hline & & $\mathrm{CN}$ & MCI & AD dem. & $\mathrm{CN}$ & MCI & AD dem. & $\mathrm{CN}$ & $\mathrm{MCI}$ & $\mathrm{AD}$ dem. \\
\hline \multirow[t]{2}{*}{${ }^{18} \mathrm{~F}-\mathrm{AV}-45$} & Low & $144(62.9)$ & $78(34.1)$ & $7(3.0)$ & $63(41.7)$ & $70(46.4)$ & $18(11.9)$ & $207(54.5)$ & 148 (38.9) & $25(6.6)$ \\
\hline & High & $49(28.7)$ & $90(52.6)$ & $32(18.7)$ & $27(13.2)$ & $90(43.9)$ & $88(42.9)$ & $76(20.2)$ & $180(47.9)$ & $120(31.9)$ \\
\hline \multirow[t]{2}{*}{$\operatorname{CSF} A \beta_{1-42}$} & Low & $47(31.1)$ & $75(49.7)$ & $29(19.2)$ & $22(11.2)$ & $90(45.7)$ & $85(43.1)$ & $69(19.8)$ & $165(47.4)$ & $114(32.8)$ \\
\hline & High & $128(59.2)$ & $82(38.0)$ & $6(2.8)$ & $58(43.9)$ & $63(47.8)$ & $11(8.3)$ & $186(53.4)$ & $145(41.7)$ & $17(4.9)$ \\
\hline \multirow[t]{2}{*}{ MRI hippocampus } & Low & $48(33.6)$ & $70(48.9)$ & $25(17.5)$ & $31(15.3)$ & 85 (41.9) & $87(42.8)$ & $79(22.8)$ & $155(44.8)$ & $112(32.4)$ \\
\hline & High & $133(61.3)$ & $76(35.0)$ & $8(3.7)$ & $55(43.0)$ & $61(47.6)$ & $12(9.4)$ & $188(54.5)$ & $137(39.7)$ & $20(5.8)$ \\
\hline \multirow[t]{2}{*}{ FDG PET } & Low & $55(31.8)$ & $85(49.1)$ & $33(19.1)$ & $26(12.2)$ & $88(41.1)$ & $100(46.7)$ & $81(20.9)$ & $173(44.7)$ & $133(34.4)$ \\
\hline & High & $143(61.1)$ & $84(35.9)$ & $7(3.0)$ & $65(45.1)$ & $74(51.4)$ & $5(3.5)$ & $208(55.0)$ & $158(41.8)$ & $12(3.2)$ \\
\hline
\end{tabular}

Low: $\leq$ median value; high: $>$ median value

Italic: normal; bold: abnormal

Median values: ${ }^{18}$ F-AV-45: 1.15 SUVR; CSF A $\beta_{1-42}: 909.5$ pg/ml; MRI hippocampus: $7.1 \mathrm{ml}$; FDG PET: 1.26 metaROI

Available data: ${ }^{18}$ F-AV-45: $n=756$; CSF A $\beta_{1-42}: n=696$; MRI hippocampus: $n=691$; FDG PET: $n=765$ 
Table 5 Results of logistic regression models exploring the association between dichotomized biomarkers (independent variables of interest) and $\mathrm{AD}$ dementia/MCI status (dependent variables of interest) stratified by Frailty Index values

\begin{tabular}{|c|c|c|c|c|c|c|}
\hline & \multicolumn{4}{|c|}{ Frailty Index } & \multicolumn{2}{|c|}{ Interaction } \\
\hline & \multicolumn{2}{|l|}{ Low } & \multicolumn{2}{|l|}{ High } & & \\
\hline AD dem. (vs. CN reference group) & OR & $95 \% \mathrm{CI}$ & OR & $95 \% \mathrm{CI}$ & OR & $95 \% \mathrm{CI}$ \\
\hline${ }^{18} \mathrm{~F}-\mathrm{AV}-45$ (high vs. low) & 19.49 & $7.69-49.41$ & 11.32 & $5.61-22.83$ & 0.58 & $0.37-0.77$ \\
\hline CSF A $\beta_{1-42}$ (low vs. high) & 15.51 & $5.90-40.79$ & 20.23 & $8.93-45.82$ & 1.30 & $0.93-1.52$ \\
\hline MRI hippocampus (low vs. high) & 19.17 & $6.98-52.64$ & 16.45 & $7.26-37.27$ & 0.86 & $0.64-0.95$ \\
\hline FDG PET (low vs. high) & 13.46 & $5.49-32.97$ & 51.90 & $18.61-144.80$ & 3.86 & $2.37-5.77$ \\
\hline MCI (vs. CN reference group) & OR & $95 \% \mathrm{CI}$ & OR & $95 \% \mathrm{CI}$ & OR & $95 \% \mathrm{CI}$ \\
\hline${ }^{18}$ F-AV-45 (high vs. low) & 4.01 & $2.51-6.40$ & 3.41 & $1.92-6.07$ & 0.85 & $0.37-0.98$ \\
\hline CSF A $\beta_{1-42}$ (low vs. high) & 2.69 & $1.68-4.30$ & 3.92 & $2.13-7.21$ & 1.46 & $0.53-1.96$ \\
\hline MRI hippocampus (low vs. high) & 3.69 & $2.19-6.21$ & 3.92 & $2.10-7.33$ & 1.06 & $0.51-1.13$ \\
\hline FDG PET (low vs. high) & 2.82 & $1.81-4.39$ & 3.28 & $1.84-5.86$ & 1.16 & $0.46-1.34$ \\
\hline
\end{tabular}

Each biomarker (categorical high vs. low or low vs. high, independent variable) is singularly included in the age-, sex-, and educationadjusted regression model to predict cognitive status (AD dem. vs. CN or MCI vs. CN, dependent variable). The population is stratified according to low or high Frailty Index values

Low: $\leq$ median value; high: > median value

Median values: ${ }^{18}$ F-AV-45: 1.15 SUVR; CSF A $\beta_{1-42}: 909.5$ pg/ml; MRI hippocampus: $7.1 \mathrm{ml}$; FDG PET: 1.26 metaROI

0.20 ) had an accuracy of nearly $70 \%$ and a sensitivity of $73.2 \%$ in discriminating participants with and without mild $\mathrm{AD}$ dementia.

\section{Discussion}

To the best of our knowledge, this study constitutes the first attempt to test the hypothesis that frailty may act as a latent factor in the relationship between $\mathrm{AD}$ candidate biomarkers and the phenotypic expression of the neurodegenerative condition. The present results confirm and extend the previous findings obtained by Wallace and colleagues based on clinicopathological data [15], that is:

(i) frailty contributes to the discrepancies between $\mathrm{AD}$ pathology and clinical manifestations; and

(ii) frailty influences the association of $\mathrm{AD}$ pathological modifications with the individual's cognitive changes (i.e., MCI and dementia).

Table 6 Results of logistic regression models exploring the association between dichotomized biomarkers (independent variables of interest) and AD dementia status (dependent variables of interest) stratified by Frailty Index values

\begin{tabular}{|c|c|c|c|c|c|c|}
\hline & \multicolumn{4}{|c|}{ Frailty Index } & \multicolumn{2}{|c|}{ Interaction } \\
\hline & Low & & High & & & \\
\hline \multirow{2}{*}{$\begin{array}{l}\text { AD dem. (vs. CN reference group) } \\
{ }^{18} \mathrm{~F}-\mathrm{AV}-45 \text { (> } 1.11 \text { SUVR) }\end{array}$} & OR & $95 \% \mathrm{CI}$ & OR & $95 \% \mathrm{CI}$ & OR & $95 \%$ CI \\
\hline & 16.36 & $6.32-42.33$ & 12.28 & $5.99-25.21$ & 0.75 & $0.51-0.90$ \\
\hline $\operatorname{CSF} \mathrm{A} \beta_{1-42}(<880 \mathrm{pg} / \mathrm{ml})$ & 8.94 & $4.13-19.34$ & 11.24 & $5.70-22.17$ & 1.26 & $0.79-1.49$ \\
\hline MRI hippocampus (<6.7 ml) & 15.54 & $6.66-36.23$ & 11.57 & $5.70-23.48$ & 0.74 & $0.50-0.90$ \\
\hline FDG PET (< 1.21 metaROI) & 17.67 & $7.69-40.63$ & 26.63 & $12.29-57.70$ & 1.51 & $1.08-1.85$ \\
\hline
\end{tabular}

Each biomarker (categorical, independent variable) is singularly included in the age-, sex-, and education-adjusted regression model to predict cognitive status (AD dem. vs. CN, dependent variable) at low and high Frailty Index values

The cut-points for each of the considered biomarkers were derived from [30-32] 
In the present analysis, approximately one out of five participants had a positive AD biomarker without presenting any cognitive disturbance and slightly less than one out of two met the $\mathrm{MCI}$ or $\mathrm{AD}$ dementia criteria in the absence of any biomarkers' change. The rate of misclassification was significantly influenced by frailty levels. The probability of being cognitively intact in the presence of a positive biomarker was higher among subjects with lower FI scores (i.e., $28.7-33.6 \%$ vs. 11.2-15.3\%). Conversely, participants with higher FI values had an increased likelihood of presenting MCI or dementia despite the normality of biomarkers (cumulative $54.9-58.3 \%$ vs. $37.1-40.7 \%$ ). Two main reflections are inspired by these findings and are aligned with the interpretations proposed by Wallace et al. [15]. First, individuals with a lower amount of health deficits seem better able to cope with the accumulation of AD neuropathology. Consistent with its definition, frailty emerges as a reduction of those reserves which enable the organism (and the brain) to tolerate the onset of pathological perturbations and modifications with limited functional consequences. The progressive accumulation of deficits lowers the threshold for $\mathrm{AD}$ pathological changes to produce cognitive deficits. Second, frailty likely concurs to the decline of cognitive functioning and the development of dementia through pathophysiological mechanisms that are not directly captured by candidate $\mathrm{AD}$ biomarkers. Frailty has already been shown to be directly associated with the main modifications of $\mathrm{AD}$, such as amyloid deposition and brain atrophy [33]. Nevertheless, it is accompanied by additional biological processes (e.g., inflammation, immunosenescence, metabolic and energetic declines, loss of proteostasis) that may synergistically contribute to the onset of dementia [34].

It is noteworthy that the individual relationships between each of the considered biomarkers and cognitive status were differently moderated by frailty. Indeed, the increase in FI weakened the association of ${ }^{18} \mathrm{~F}-\mathrm{AV}-45$ PET status and MRI hippocampal volume with $\mathrm{AD}$ dementia. On the other hand, it strengthened the relationship between FDG PET status and AD dementia. In other words, the likelihood that a positive amyloid PET scan and an MRIbased evidence of hippocampus atrophy manifested with $\mathrm{AD}$ dementia was higher among participants with lower frailty levels. Conversely, higher frailty scores markedly increased the probability that brain hypometabolism resulted in an overt dementia condition. These findings suggest that the pathogenic contribution of some of the $\mathrm{AD}$ pathophysiological processes (i.e., amyloid deposition, neuronal loss) is significantly influenced by the background noise of the organism's biology [35]. Therefore, the interpretation of the changes affecting the relative biomarkers may be biased by the individual's biological complexity. Consequently, the current research approach (and the proposed diagnostic framework [18]) based on a bivariate conception of the biomarker is probably inadequate and results in a reductionistic picture of a complex phenomenon. The introduction of a third dimension, such as frailty, may instead enhance the understanding of the multifaceted pathways leading to neurodegeneration, their link with phenotypic manifestations and clinical diagnoses, and possibly their suitability as research targets. In this regard, it should be observed that tau deposition was not affected by frailty as suggested by the detection of similar CSF levels of T-tau and ${ }^{181} \mathrm{P}$-tau in the two FI groups (Table 1). Thus, the cascade of molecular events leading to the formation of neurofibrillary tangles may theoretically be regarded as more specific of neurodegeneration, including $\mathrm{AD}$, compared to other pathways and abnormalities that emerged as likely more consistently shared with the multisystemic aging process. This latter result is in line with the findings by Wallace and colleagues who observed that the interaction between frailty and dementia status was essentially driven by amyloid rather than tau pathology [15]. Interestingly, the findings on the interaction between frailty and amyloid deposition were not fully concordant when it was measured in terms of florbetapir uptake (significant interaction with frailty) or CSF $\mathrm{A} \beta_{1-42}$ levels (non-significant interaction with frailty) (Table 5). However, it is noteworthy that a modest agreement between the two biomarkers was observed in the sample (kappa $=0.66 ; p<0.001$ ), with nearly $17 \%$ of subjects being discordantly classified based on the two diagnostic procedures.

Overall, the present findings may have important practical implications. The routine adoption of a versatile measure such as the FI may provide useful, additional information when exploring the clinical expression of $\mathrm{AD}$ and assessing the presence of cognitive disorders in older individuals. This may also be the case in hyper-selected populations of participants in research protocols where monodimensional assessments may fail to adequately discriminate between different clinical and biological profiles. Accordingly, the use of the FI may consent to render more homogeneous the study samples in terms of clinical and biological complexity, thus increasing the external validity of the observed findings [13]. Also, this tool may support a person-centered reading of the results from biomarker assessments, thus attributing the proper weight to the biological abnormalities documented in a given individual. 
This may ultimately sustain personalized therapeutic approaches.

This study has several limitations to be mentioned. The cross-sectional design impedes to infer about causality in the observed relationship between frailty, $\mathrm{AD}$ pathology, and cognitive status. Longitudinal data on the modifications of the factors are fundamental to better elucidate their interaction. The analysis was conducted in a highly selected sample of subjects that are probably not representative of the "real world" population of older people with intact or declining cognition [36]. Individuals with unstable or severe medical conditions (e.g., stroke, cancer, health failure) and MRI evidence of relevant brain vascular pathology (i.e., infarctions, multiple and/or strategic lacunes) were, in fact, not considered for participation in the ADNI study. This has probably attenuated the overall frailty levels of the considered subjects and limited the possibility of properly accounting for the effect of additional pathophysiological mechanisms (e.g., vascular pathology) that have been frequently associated with dementia and commonly contribute to mixed neuropathologies [37]. The decision to base the present analyses on the median values of the considered biomarkers rather than on clinically validated cut-points has potentially contributed to misclassify a non-negligible share of participants. Nevertheless, this approach was motivated by the fact that, in the ADNI study, multiple cutoffs have been calculated for each biomarker thus making it arbitrary the choice on which of them to rely on. Anyway, the use of ADNIderived cutoff values in sensitivity analyses did not substantially change the findings.

'In conclusion, the present study confirms that frailty influences the neuropathophysiology and clinical expression of AD. The age-related accumulation of health deficits significantly moderates the association between several pathways implicated in the pathogenesis of the disease and its phenotypic manifestations. Moreover, it affects the individual threshold at which neuropathological changes result in clinical impairments and diagnostic entities. $\mathrm{AD}$ and dementia should increasingly be conceived as "complex diseases of aging" [15], determined by multiple, simultaneous, and interacting pathophysiological processes, thus abandoning one-fits-all and reductionist interpretations. In this alternative framework, the adoption of the frailty construct may improve our comprehension of the biological modifications contributing to dementia, consent to better interpret the findings of diagnostic procedures, and possibly better calibrate therapeutic targets and interventions.

Acknowledgments Data collection and sharing for this project was funded by the Alzheimer's Disease Neuroimaging Initiative (ADNI) (National Institutes of Health Grant U01 AG024904) and DOD ADNI (Department of Defense award number W81XWH12-2-0012). ADNI is funded by the National Institute on Aging, by the National Institute of Biomedical Imaging and Bioengineering, and through generous contributions from the following: AbbVie, Alzheimer's Association; Alzheimer's Drug Discovery Foundation; Araclon Biotech; BioClinica, Inc.; Biogen; BristolMyers Squibb Company; CereSpir, Inc.; Cogstate; Eisai Inc.; Elan Pharmaceuticals, Inc.; Eli Lilly and Company; EuroImmun; F. Hoffmann-La Roche Ltd and its affiliated company Genentech, Inc.; Fujirebio; GE Healthcare; IXICO Ltd.; Janssen Alzheimer Immunotherapy Research \& Development, LLC.; Johnson \& Johnson Pharmaceutical Research \& Development LLC.; Lumosity; Lundbeck; Merck \& Co., Inc.; Meso Scale Diagnostics, LLC.; NeuroRx Research; Neurotrack Technologies; Novartis Pharmaceuticals Corporation; Pfizer Inc.; Piramal Imaging; Servier; Takeda Pharmaceutical Company; and Transition Therapeutics. The Canadian Institutes of Health Research is providing funds to support ADNI clinical sites in Canada. Private sector contributions are facilitated by the Foundation for the National Institutes of Health (www.fnih.org). The grantee organization is the Northern California Institute for Research and Education, and the study is coordinated by the Alzheimer's Therapeutic Research Institute at the University of Southern California. ADNI data are disseminated by the Laboratory for Neuro Imaging at the University of Southern California.

Authors' contributions M.Ca.: conception and design of the study, data analysis, writing of the manuscript;

I.A.: analysis of data, drafting of the manuscript;

I.B.: analysis of data;

A.A.: drafting of the manuscript;

D.G.: drafting of the manuscript;

N.V.: analysis of data, drafting of the manuscript;

M.D.: analysis of data, drafting of the manuscript;

M.Ce.: conception and design of the study, drafting of the manuscript;

G.B.: conception and design of the study, drafting of the manuscript.

Funding Open access funding provided by Università degli Studi di Roma La Sapienza within the CRUI-CARE Agreement. This study was partially supported by the grant POR (Operative Program Lazio Region, Italy) FESR (European Program Regional Development) 2014 - 2020. Public Notice "LIFE 2020", MODIAG Project. This work was also supported (in part) by Fondo Ordinario Enti (FOE D.M 865/2019) funds in the framework of a collaboration agreement between the Italian National Research Council and EBRI (2019-2021).Data availability Data used in preparation of this article were obtained from the Alzheimer's Disease Neuroimaging Initiative (ADNI) database (adni.loni.usc.edu). 


\section{Compliance with ethical standards}

Competing interests The authors have no conflicts of interest to disclose for the present study. Marco Canevelli is supported by a research grant of the Italian Ministry of Health (GR-201602364975) for the project "Dementia in immigrants and ethnic minorities living in Italy: clinical-epidemiological aspects and public health perspectives" (ImmiDem). Matteo Cesari has received honoraria for presentations at scientific meetings and/or research funding from Nestlé and Pfizer. He is involved in the coordination of an Innovative Medicines Initiative-funded project (including partners from the European Federation Pharmaceutical Industries and Associates [Sanofi, Novartis, Servier, GSK, Lilly]).

Ethics approval and consent to participate As per ADNI protocols, all procedures performed in studies involving human participants were in accordance with the ethical standards of the institutional and/or national research committee and with the 1964 Helsinki declaration and its later amendments or comparable ethical standards. More details can be found at adni.loni.usc.edu. The study was approved by the institutional review boards of all the participating institutions, and informed written consent was obtained from all participants at each site. This article does not contain any studies with human participants performed by any of the authors.

Consent for publication Consent for publication has been granted by ADNI administrators.

Open Access This article is licensed under a Creative Commons Attribution 4.0 International License, which permits use, sharing, adaptation, distribution and reproduction in any medium or format, as long as you give appropriate credit to the original author(s) and the source, provide a link to the Creative Commons licence, and indicate if changes were made. The images or other third party material in this article are included in the article's Creative Commons licence, unless indicated otherwise in a credit line to the material. If material is not included in the article's Creative Commons licence and your intended use is not permitted by statutory regulation or exceeds the permitted use, you will need to obtain permission directly from the copyright holder. To view a copy of this licence, visit http://creativecommons.org/licenses/by/4.0/.

\section{References}

1. Espay AJ, Vizcarra JA, Marsili L, et al. Revisiting protein aggregation as pathogenic in sporadic Parkinson and Alzheimer diseases. Neurology. 2019;92(7):329-37. https://doi.org/10.1212/WNL.0000000000006926.

2. Illán-Gala I, Pegueroles J, Montal V, et al. Challenges associated with biomarker-based classification systems for Alzheimer's disease. Alzheimers Dement Diagn Assess Dis Monit. 2018;10:346-57. https://doi.org/10.1016/j. dadm.2018.03.004.
3. Clegg A, Young J, Iliffe S, et al. Frailty in elderly people. Lancet. 2013;381(9868):752-62. https://doi.org/10.1016 /S0140-6736(12)62167-9.

4. Morley JE, Vellas B, van Kan GA, et al. Frailty consensus: a call to action. J Am Med Dir Assoc. 2013;14(6):392-7. https://doi.org/10.1016/j.jamda.2013.03.022.

5. Hoogendijk EO, Afilalo J, Ensrud KE, et al. Frailty: implications for clinical practice and public health. Lancet. 2019;394(10206):1365-75. https://doi.org/10.1016/S01406736(19)31786-6.

6. Rockwood K, Howlett SE. Age-related deficit accumulation and the diseases of ageing. Mech Ageing Dev. 2019;180: 107-16. https://doi.org/10.1016/j.mad.2019.04.005.

7. Mitnitski AB, Mogilner AJ, Rockwood K. Accumulation of deficits as a proxy measure of aging. ScientificWorldJournal. 2001;1:323-36. https://doi. org/10.1100/tsw.2001.58.

8. Rogers NT, Steptoe A, Cadar D. Frailty is an independent predictor of incident dementia: evidence from the English Longitudinal Study of Ageing. Sci Rep. 2017;7. https://doi. org/10.1038/s41598-017-16104-y.

9. Song X, Mitnitski A, Rockwood K. Nontraditional risk factors combine to predict Alzheimer disease and dementia. Neurology. 2011;77(3):227-34. https://doi.org/10.1212 /WNL.0b013e318225c6bc.

10. Kelaiditi E, Andrieu S, Cantet C, et al. Frailty Index and incident mortality, hospitalization, and institutionalization in Alzheimer's disease: data from the ICTUS study. J Gerontol A Biol Sci Med Sci. 2015;71(4):543-8. https://doi. org/10.1093/gerona/glv137.

11. Kelaiditi E, Canevelli M, Andrieu S, et al. Frailty Index and cognitive decline in Alzheimer's disease: data from the Impact of Cholinergic Treatment USe study. J Am Geriatr Soc. 2016;64(6):1165-70. https://doi.org/10.1111 /jgs.13956.

12. Trebbastoni A, Canevelli M, D'Antonio F, et al. The impact of frailty on the risk of conversion from mild cognitive impairment to Alzheimer's disease: evidences from a 5year observational study. Front Med. 2017;4. https://doi. org/10.3389/fmed.2017.00178.

13. Canevelli M, Trebbastoni A, Quarata F, et al. External validity of randomized controlled trials on Alzheimer's disease: the biases of frailty and biological aging. Front Neurol. 2017;8:628. https://doi.org/10.3389/fneur.2017.00628.

14. Maltais M, De Souto Barreto P, Hooper C, et al. Association between brain $\beta$-amyloid and frailty in older adults. $\mathrm{J}$ Gerontol Ser A. 2019;74(11):1747-52. https://doi. org/10.1093/gerona/glz009.

15. Wallace LMK, Theou O, Godin J, et al. Investigation of frailty as a moderator of the relationship between neuropathology and dementia in Alzheimer's disease: a crosssectional analysis of data from the Rush Memory and Aging Project. Lancet Neurol. 2019;18(2):177-84. https://doi.org/10.1016/S1474-4422(18)30371-5.

16. Jack CR, Albert MS, Knopman DS, et al. Introduction to the recommendations from the National Institute on AgingAlzheimer's Association workgroups on diagnostic guidelines for Alzheimer's disease. Alzheimers Dement. 2011;7(3):257-62. https://doi.org/10.1016/j. jalz.2011.03.004. 
17. Blennow K. Biomarkers in Alzheimer's disease drug development. Nat Med. 2010;16(11):1218-22. https://doi. org/10.1038/nm.2221.

18. Jack CR, Bennett DA, Blennow K, et al. NIA-AA research framework: toward a biological definition of Alzheimer's disease. Alzheimers Dement. 2018;14(4):535-62. https://doi.org/10.1016/j.jalz.2018.02.018.

19. Olsson B, Lautner R, Andreasson U, et al. CSF and blood biomarkers for the diagnosis of Alzheimer's disease: a systematic review and meta-analysis. Lancet Neurol. 2016;15(7):673-84. https://doi.org/10.1016/S1474-4422(16 00070-3.

20. Petersen RC, Smith GE, Waring SC, et al. Mild cognitive impairment: clinical characterization and outcome. Arch Neurol. 1999;56(3):303-8.

21. Petersen RC, Aisen PS, Beckett LA, et al. Alzheimer's Disease Neuroimaging Initiative (ADNI): clinical characterization. Neurology. 2010;74(3):201-9. https://doi. org/10.1212/WNL.0b013e3181cb3e25.

22. Wechsler DA. Wechsler Memory Scale-Revised. New York Psychological Corporation 1987.

23. McKhann G, Drachman D, Folstein M, et al. Clinical diagnosis of Alzheimer's disease: report of the NINCDSADRDA Work Group under the auspices of Department of Health and Human Services Task Force on Alzheimer's Disease. Neurology. 1984;34(7):939-44.

24. Jack CR, Barnes J, Bernstein MA, et al. Magnetic resonance imaging in ADNI. Alzheimers Dement. 2015;11(7):740-56. https://doi.org/10.1016/j.jalz.2015.05.002.

25. Jagust WJ, Landau SM, Koeppe RA, et al. The Alzheimer's Disease Neuroimaging Initiative 2 PET Core: 2015. Alzheimers Dement. 2015;11(7):757-71. https://doi. org/10.1016/j.jalz.2015.05.001.

26. Landau SM, Fero A, Baker SL, et al. Measurement of longitudinal $\beta$-amyloid change with $18 \mathrm{~F}$-florbetapir PET and standardized uptake value ratios. J Nucl Med. 2015;56(4):567-74. https://doi.org/10.2967 /jnumed.114.148981.

27. Shaw LM, Vanderstichele H, Knapik-Czajka M, et al. Cerebrospinal fluid biomarker signature in Alzheimer's disease neuroimaging initiative subjects. Ann Neurol. 2009;65(4):403-13. https://doi.org/10.1002/ana.21610.

28. Searle SD, Mitnitski A, Gahbauer EA, et al. A standard procedure for creating a Frailty Index. BMC Geriatr. 2008;8:24. https://doi.org/10.1186/1471-2318-8-24.
29. Hwang SJ, Beaty TH, Liang KY, et al. Minimum sample size estimation to detect gene-environment interaction in case-control designs. Am J Epidemiol. 1994;140(11): 1029-37. https://doi.org/10.1093/oxfordjournals.aje. a117193.

30. Hansson O, Seibyl J, Stomrud E, et al. CSF biomarkers of Alzheimer's disease concord with amyloid- $\beta$ PET and predict clinical progression: a study of fully automated immunoassays in BioFINDER and ADNI cohorts. Alzheimers Dement. 2018;14(11):1470-81. https://doi.org/10.1016/j. jalz.2018.01.010.

31. Landau SM, Mintun MA, Joshi AD, et al. Amyloid deposition, hypometabolism, and longitudinal cognitive decline. Ann Neurol. 2012;72(4):578-86. https://doi.org/10.1002 /ana.23650.

32. Ou Y-N, Xu W, Li J-Q, et al. FDG-PET as an independent biomarker for Alzheimer's biological diagnosis: a longitudinal study. Alzheimers Res Ther. 2019;11(1):57. https://doi. org/10.1186/s13195-019-0512-1.

33. Wallace L, Theou O, Rockwood K, Andrew MK. Relationship between frailty and Alzheimer's disease biomarkers: a scoping review. Alzheimers Dement Diagn Assess Dis Monit. 2018;10:394-401. https://doi. org/10.1016/j.dadm.2018.05.002.

34. Bisset ES, Howlett SE. The biology of frailty in humans and animals: understanding frailty and promoting translation. Aging Med. 2019;2(1):27-34. https://doi.org/10.1002 /agm2.12058.

35. Canevelli M, Cesari M, Raganato R, et al. Role of frailty in the assessment of cognitive functioning. Mech Ageing Dev. $2019 ; 181: 42-6$. https://doi.org/10.1016/j. $\operatorname{mad} .2019 .111122$.

36. Weiner MW, Aisen PS, Jack CR, et al. The Alzheimer's Disease Neuroimaging Initiative: progress report and future plans. Alzheimers Dement. 2010;6(3):202-11. e7. https://doi.org/10.1016/j.jalz.2010.03.007.

37. Boyle PA, Yu L, Wilson RS, Leurgans SE, et al. Personspecific contribution of neuropathologies to cognitive loss in old age. Ann Neurol. 2018;83(1):74-83. https://doi. org/10.1002/ana.25123.

Publisher's note Springer Nature remains neutral with regard to jurisdictional claims in published maps and institutional affiliations. 